\title{
Repetition of FDG PET study in the same day after appropriate patient preparation revealed two new cancer localizations:
}

\section{a case report}

Andrea d'Amico, Cezary Przeorek, Maria Turska-d'Amico

Maria Skłodowska-Curie Memorial Cancer Center and Institute of Oncology, Gliwice branch

[Received 9 I 2013; Accepted 10 VI 2013]

\section{Abstract}

A positron emission tomography scan with fluorodeoxyglucose can be affected by several factors. Skeletal muscle activation and physiological presence of radioactivity in urine frequently cause difficulties in images interpretation. We report a case of a patient with a non-operable left lung cancer, who was scheduled for FDG PET examination for radiotherapy planning purposes. In the first scan performed in the morning both elevated muscular and urinary uptake were present. For this reason another examination was performed on the same day. A new dose of radiopharmaceutical was given five hours after the first FDG injection and the patient was instructed to drink a large quantity of water in the meantime. The second PET scan clearly revealed two new lesions not visible at the time of the first examination: a synchronous bladder cancer, previously not known, and a mediastinal metastasis of the primary lung cancer. This case emphasizes the importance of correct patient preparation and shows the possibility to repeat PET examination on the same day.

KEY words: FDG PET, patient management, hydration

Nuclear Med Rev 2013; 16, 2: 88-90

\section{Introduction}

The quality of a positron emission tomography scan with 18F-fluorodeoxyglucose injection (FDG PET) depends highly on

Correspondence to: Andrea d'Amico

Maria Skłodowska-Curie Memorial Cancer Center and Institute of Oncology, Gliwice branch

E-mail: adamico@io.gliwice.pl patient preparation. Meal consumption before FDG PET examination is followed by insulin secretion causing extensive uptake of radiopharmaceutical in skeletal muscle. In consequence, visual interpretation is negatively affected. Moreover, the evaluation of the bladder wall is usually precluded by physiological FDG excretion in urine if a patient has not undergone adequate hydration i.e. has neither drunk water nor had intravenous infusion. We report a PET-CT case of a patient with lung cancer. Due to the low quality of the first PET scan, the examination was redone after only 5 hours. This time two important finds were revealed, namely: synchronous bladder cancer and suspect lymph node metastases. They were not visible in the previous scan.

\section{Case report}

A 79-year-old male with inoperable non-small cell lung cancer of the upper left lobe underwent palliative radiotherapy treatment in December 2006. Left hilar adenopathy was detected in a follow-up CT examination in March 2012. The patient's condition deteriorated further during the subsequent months and he was referred to FDG PET-CT examination in order to possibly schedule second radiotherapy treatment. That PET-CT was performed in September 2012.

Due to the patient's clinical condition as well as his age, the PET scan was scheduled as the first in the morning. At the patient's arrival his glycemia was measured: $118 \mathrm{mg} / \mathrm{ml}$, and he was given an injection of $320 \mathrm{MBq} 18 \mathrm{~F}$-Fluorodeoxygtlucose at 8:10. The PET-CT scan was performed at 9:07 with the use of Siemens mCT machine. It was performed from the skull base to the thigh with time acquisition 120" per bed position. No contrast medium was given for CT.

Immediately after the image reconstruction, strong FDG uptake in skeletal muscle was noticed on MIP projection (Fig. 1A). The patient admitted that despite the instruction, he had had light breakfast about 1 hour before the examination. Even though pathological FDG uptake was observed in the left hilum, the scan quality was generally evaluated as non-acceptable.

Taking into account the patient's disagreement to re-schedule the examination, the nuclear medicine specialist decided to redo the scan in five hours' time. The patient was instructed to fast and to drink at least 1 liter of water. 

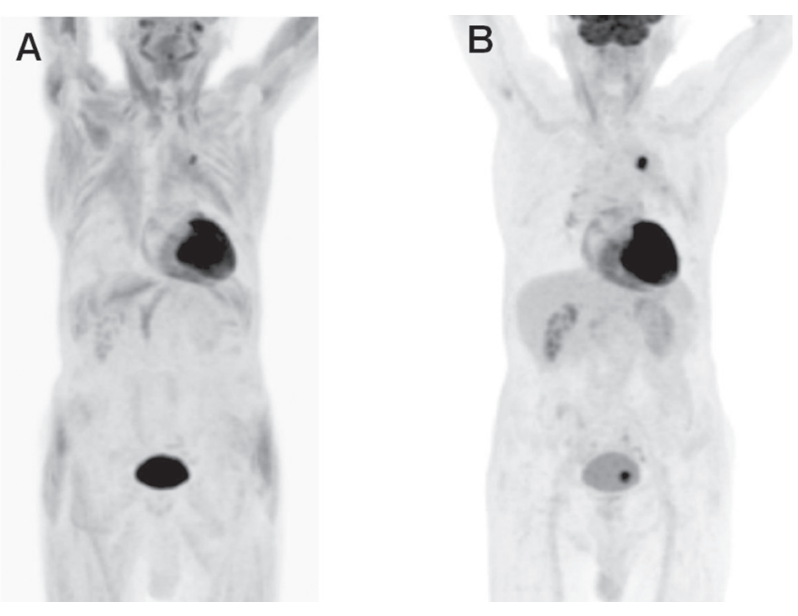

Figure 1. MIP projection of the first FDG PET scan (A) and the second one $(B)$ performed 5 hours later on the same day

The second injection of $295 \mathrm{MBq}$ of 18-F FDG was performed at 13:45. At this time the glycemic level had the value of $96 \mathrm{mg} / \mathrm{dl}$. The patient underwent the second PET-CT scan at 14.38 on the same scanner. The image quality was evaluated as good (Fig. 1B).
The pathological hot spot on the left pulmonary hilum was confirmed. After that, faint uptake on a roundish right pre-tracheal lymph node, not evident on the first scan, became clearly visible (Fig. 2)

Surprisingly, intense pathological FDG uptake, previously completely undetectable, was revealed on the bladder wall. Histopathological examination confirmed the presence of bladder cancer. A radiologically nonspecific thickening of the left posterior bladder wall was visible in the CT images in both examinations (Fig. 3).

\section{Discussion}

Hydration or alternatively diuretic administration allows diluting the radioactivity concentration in the urinary tract as they increase urine volume. This points to the necessity of implementing several protocols for patient preparation, which can significantly improve the detection rate of primary bladder malignancy [5-7].

Furthermore, increased insulin secretion, due to a non-fasting state, interferes with FDG uptake in malignant lesions by increasing muscular and cardiac uptake, which leads to altered radiotracer biodistribution. The increased avidity of insulin-stimulated muscular masses weakens the FDG uptake in cancer sites. Moreover, in small lesions - in which the partial volume effect plays a major role - the reduced tumor/background ratio can lead to a false negative scan [8-10].

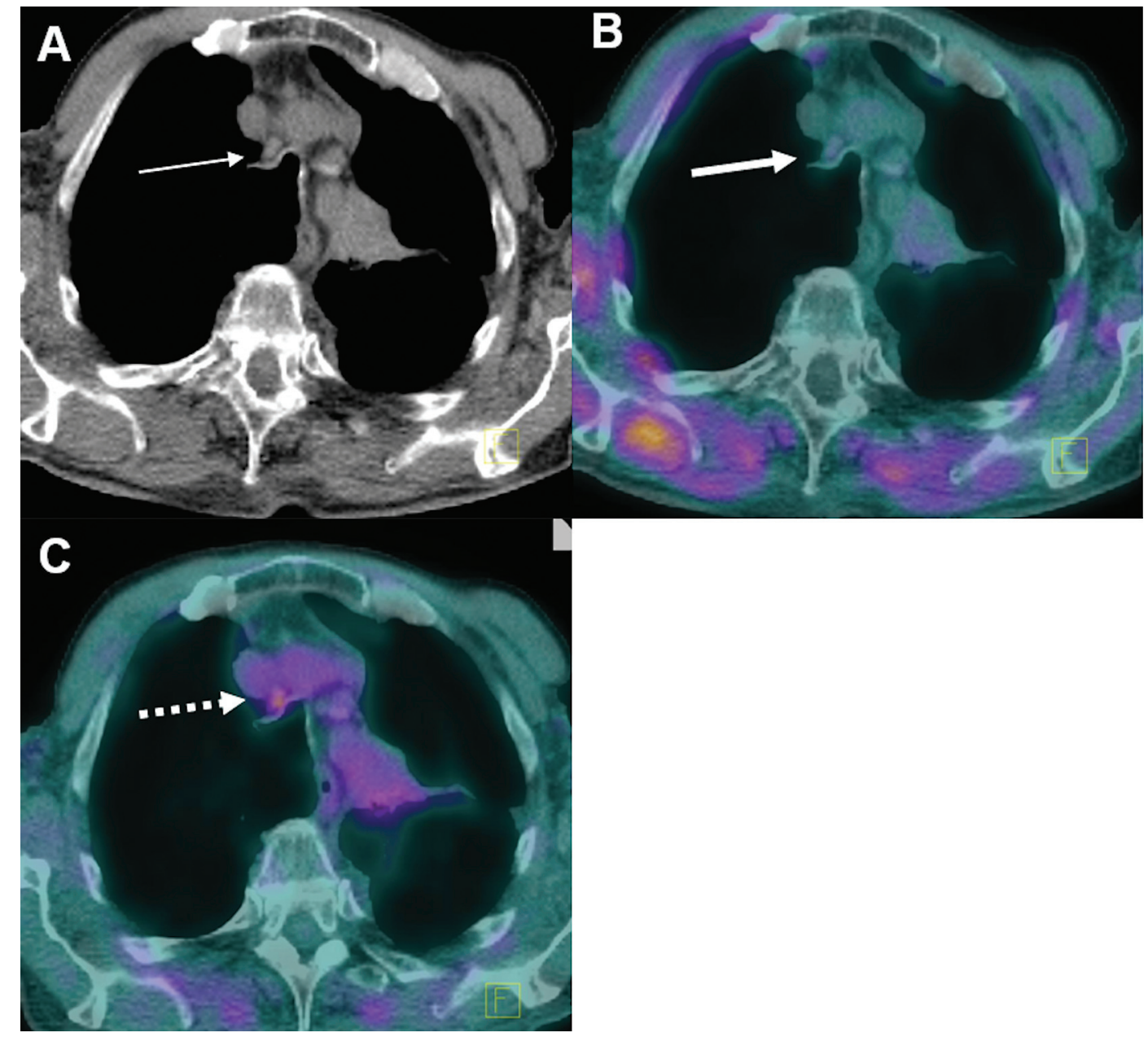

Figure 2. A non enlarged right paratracheal lymph node visible on CT (A, thin arrow) has no FDG uptake at first FDG PET scan (B, thick arrow) but showed a pathological activity at second scan (C, dotted arrow) 


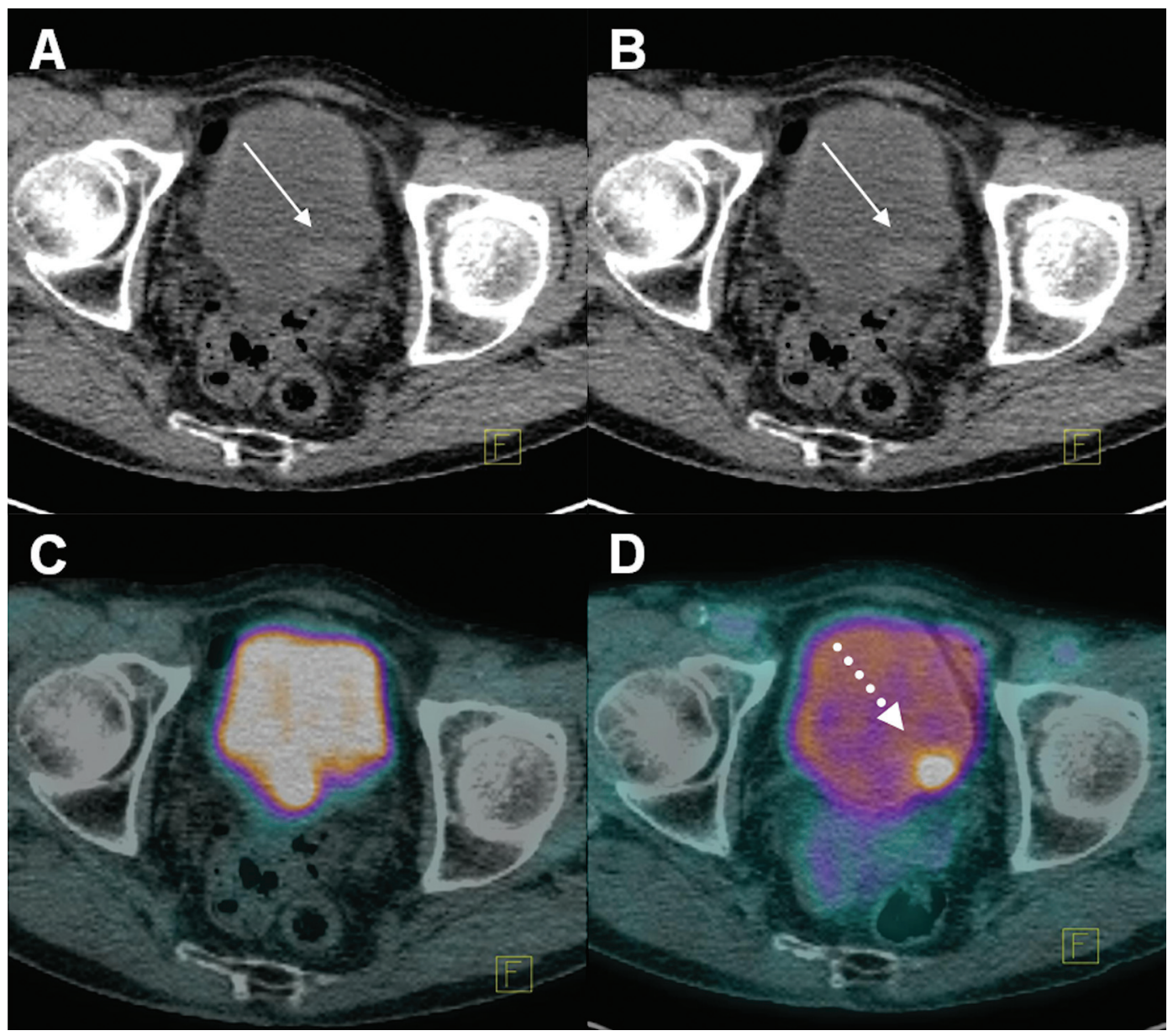

Figure 3. Bladder wall thickening is visible on CT images of both examinations (A, B, thin arrows). Urinary radioactivity masked this finding at first scan (C), conversely an evident pathological FDG uptake is visible on this site after adequate hydration (D, dotted arrow)

\section{Conclusion}

The implementation of appropriate patient preparation procedures for all PET patients plays a pivotal role for obtaining the highest possible quality of the scan. It enhances the detection rate of small metastases and increases the possibility to visualize a metasynchronous malignancy.

\section{References}

1. Nakatani K, Nakamoto Y, Togashi K. Risk factors for extensive skeletal muscle uptake in oncologic FDG-PET/CT for patients undergoing a 4-h fast. Nucl Med Ommun 2012; 33: 648-655.

2. Chin BB, Green ED, Turkington TG, Hawk TC, Coleman RE. Increasing uptake time in FDG-PET: standardized uptake values in normal tissues at 1 versus 3 h. Mol Imaging Biol 2009; 11: 118-122.

3. Reinking MF, Osman MM. Prospective evaluation of physiologic uptake detected with true whole-body 18 F-FDG PET/CT in healthy subjects. J Nucl Med Technol 2009; 37: 31-37.

4. Metser U, Even-Sapir E. Increased (18)F-fluorodeoxyglucose uptake in benign, nonphysiologic lesions found on whole-body positron emis sion tomography/computed tomography (PET/CT): accumulated data from four years of experience with PET/CT. Semin Nucl Med 2007; 37: 206-222

5. Yang Z, Cheng J, Pan L et al. Is whole-body fluorine-18 fluorodeoxyglucose PET/CT plus additional pelvic images (oral hydration-voiding-refilling) useful for detecting recurrent bladder cancer? Ann Nucl Med 2012; 26: 571-577.

6. Anjos DA, Etchebehere EC, Ramos CD, Santos AO, Albertotti C, Camargo EE. 18F-FDG PET/CT delayed images after diuretic for restaging invasive bladder cancer. J Nucl Med 2007; 48: 764-770.

7. Ceriani L, Suriano S, Ruberto T, Giovanella L. Could different hydration protocols affect the quality of 18F-FDG PET/CT images? J Nucl Med Technol 2011; 39: 77-82

8. Vriens D, Visser EP, de Geus-Oei LF, Oyen WJ. Methodological considerations in quantification of oncological FDG PET studies. Eur J Nucl Med Mol Imaging 2010; 37: 1408-1025.

9. Lee $\mathrm{KH}, \mathrm{Ko} \mathrm{BH}$, Paik JY et al. Effects of anesthetic agents and fasting duration on 18F-FDG biodistribution and insulin levels in tumor-bearing mice. J Nucl Med 2005; 46: 1531-1536.

10. Lindholm P, Minn H, Leskinen-Kallio S, Bergman J, Ruotsalainen U, Joensuu H. Influence of the blood glucose concentration on FDG uptake in cancer a PET study. J Nucl Med 1993; 34: 1-6. 